\title{
ASOCIACIÓN ENTRE FACTORES NEUROPSICOLÓGICOS, PROCESOS COGNITIVOS Y NIVELES DE LECTURA EN NIÑOS DE DIFERENTE NIVEL SOCIOECONÓMICO DEL CALLAO
}

\author{
ASSOCIATION BETWEEN NEUROPSYCHOLOGICAL FACTORS, COGNITIVE \\ PROCESSES AND READING LEVELS IN CHILDREN OF DIFFERENT \\ SOCIOECONOMIC STATUS CALLAO \\ Ricardo Canales G.* \\ Universidad Nacional Mayor de San Marcos, Perú \\ (RECIBIDO 7-5-2013, ACEPTADO 20-7-2013)
}

\section{RESUMEN}

La presente investigación buscó aportar en el conocimiento de la posible asociación entre factores neuropsicológicos, procesos cognitivos y procesos de la lectura, en niños de dos niveles socioeconómicos en el Callao, Perú.

Se usó un diseño descriptivo correlacional examinándose a 60 niños de escuela privada y pública de niveles socioeconómicos medio alto B y muy bajo E; con la Prueba neuropsicológica infantil de Quintanar- Solovieva, el WISC- IV (test de inteligencia de Weschler) y la Prueba de evaluación de lectura PROLEC- R de Cuetos.

Al final, se halló asociación de diverso tipo entre diversos factores neuropsicológicos, procesos cognitivos y procesos de la lectura en los niños de la muestra; así como un rendimiento diferenciado en procesos cognitivos e inteligencia, y en procesos de la lectura a favor de los niños del nivel socioeconómico medio alto $\mathrm{B}$.

Palabras clave: factores neuropsicológicos, lectura, inteligencia, nivel socioeconómico

\begin{abstract}
This research sought to contribute to the knowledge of the possible association between neuropsychological factors, cognitive processes and reading processes in children from two socioeconomic levels in Callao, Peru.

We used a descriptive correlational design examining 60 children from private and public schools from medium/high socio-economic level (B) and low (E), with the child neuropsychological test Solovieva Quintanar, the WISC-IV (Wechsler intelligence test ) and reading Achievement Test PROLEC-R by Cuetos.
\end{abstract}


ASOCIACIÓN ENTRE FACTORES NEUROPSICOLÓGICOS, PROCESOS COGNITIVOS Y NIVELES DE LECTURA...

Finally, we found association between various types of neuropsychological factors, cognitive processes and reading processes in examinated children, differential performance in cognitive processes and intelligence and process of reading for children from medium/high socioeconomic level (B).

Keywords: neuropsychological factors, reading, intelligence, socio-economic level

\section{INTRODUCCIÓN}

En el Perú, existe un grave problema en relación a la lectura. Los últimos lugares alcanzados por los alumnos en las diversas evaluaciones internacionales (Laboratorio Latinoamericano de Evaluación de la Calidad Educativa, 1996, 2006; PISA, 2001, 2009, Banco Mundial, 2007, etc.); así como los deficientes rendimientos en esa materia tal como lo muestran las evaluaciones nacionales realizadas por el propio Ministerio de Educación del Perú, lo confirman.

Desde la óptica de la psicología educativa, hace algunos años se vienen estudiando los problemas del aprendizaje de la lectura y la escritura. Se ha avanzado en detectar los factores cognitivos y psicolinguísticos que intervendrían en dichas deficiencias, mostrándose relevantes la conciencia fonológica, la memoria verbal operativa y la función nominativa del lenguaje (Vellutino, 1987, Domínguez, 1999, Bravo y Orellana, 1999).

Dicha orientación se ha visto confirmada en el país, con investigaciones como las de Canales y Velarde (2008) que en un estudio realizado en niños(as) de 8 años del tercer grado de primaria del Callao pertenecientes a dos niveles socioeconómicos, para encontrar la relación entre la conciencia fonológica y el nivel de decodificación lectora y comprensión lectora; hallaron correlación muy significativa entre el nivel de conciencia fonémica con el nivel de decodificación. Luego, Velarde (2009) demostró que aplicando un Programa de Habilidades metalingüísticas a niños de 8 a 10 años, de $3 .{ }^{\text {er }}$ y $4 .{ }^{\text {to }}$ grado de primaria del Callao, mejoraban en su nivel de rendimiento lector.

Pero, se requería avanzar hacia una mejor precisión de las variables internas que tendrían relación con los procesos de la lectura.

En ese sentido, en el presente estudio, se desarrolla una aproximación al problema desde el campo de la psicología cognitiva y la neuropsicología infantil. En particular, desde el punto de vista de la neuropsicología, en la línea de la escuela histórico-cultural de Vygostki y Luria, en el proceso de adquisición y desarrollo del lenguaje del niño -oral y escrito- es de suma importancia estudiar los factores neuropsicológicos, tal y como se desarrollan en el cerebro y mente de los niños.

En tal sentido, nos planteamos la siguiente pregunta de investigación:

¿Existe asociación entre factores neuropsicológicos, procesos cognitivos y procesos psicológicos de la lectura en niños pertenecientes al nivel socioeconómico medio-alto y nivel socioeconómico bajo del Callao? Si ello es así, ¿cómo se presenta dicha asociación? 
Los objetivos de la investigación fueron:

\section{Objetivo general:}

- Determinar la existencia de asociación, y sus características, entre los factores neuropsicológicos, procesos cognitivos y procesos psicológicos de lectura, en los niños del nivel socioeconómico bajo y del nivel socioeconómico medio-alto.

\section{Objetivos específicos:}

- Determinar el grado de desarrollo de los factores neuropsicológicos, cognitivos y procesos del aprendizaje de la lectura en los niños del nivel socioeconómico bajo y del nivel socioeconómico medio-alto.

- Establecer el grado de asociación entre el desarrollo los factores neuropsicológicos, cognitivos y de aprendizaje de la lectura en los niños del nivel socioeconómico bajo y del nivel socioeconómico medio-alto.

- Determinar las características de la asociación entre los factores neuropsicológicos, cognitivos y de procesos de la lectura en los niños del nivel socioeconómico bajo y del nivel socioeconómico medio-alto.

- Identificar el nivel de desarrollo de los factores neuropsicológicos y cognitivos, así como de procesos psicológicos de la lectura en los niños de los dos niveles socioeconómicos.

Nuestro estudio se inscribe en la línea de investigación sobre lectura y aspectos neuropsicológicos. Entre dichos estudios tenemos los siguientes:

Cuetos, Rodríguez y Ruano (1996) investigaron respecto a los procesos psicológicos que intervienen en el aprendizaje de la lectura, y publicaron una batería para examinar en detalle los procesos perceptivos, léxicos, sintácticos y semánticos, que intervienen en dicho aprendizaje.

Quintanar et al. (2004), siguiendo la orientación histórico-cultural, encontró que los niños pueden presentar dificultades de aprendizaje escolar debido o bien a un problema en el desarrollo del sistema nervioso central o a un problema en la interacción con el medio social. Lázaro (2001) estudió el rendimiento en tareas neuropsicológicas de niños de $1 .{ }^{\text {er }}$ a $6 .{ }^{\text {to }}$ grado de primaria en Puebla, zonas urbana y rural y buscó establecer la asociación con el nivel socioeconómico. Halló que los niños de zona rural muestran déficits en procesos de regulación y control: $85 \%$ de fallas, análisis y síntesis espacial: 100\%, organización cinética del movimiento: $90 \%$.

En el Perú, Velasco y Castro (2002) estudiaron funciones neuropsicológicas básicas a 20 niños con epilepsia y problemas de aprendizaje, en el Instituto de Ciencias Neurológicas de Lima, hallándose que dichos niños, en especial los varones, presentaban deficiencias marcadas en la atención, así como alteraciones perceptivas.

Velarde (2001) comparó el rendimiento de niños de 3 er $^{\text {er }}$ grado de primaria de dos niveles socioeconómicos distintos, a nivel de conciencia fonológica, decodificación y comprensión lectora. En el nivel socioeconómico bajo, se halló alta correlación entre conciencia fonológica y decodificación lectora (pero no con la comprensión), 
y en el nivel socioeconómico medio, se halló alta correlación entre conciencia fonológica y decodificación lectora, así como también con la comprensión lectora.

\section{Hipótesis general:}

Existe asociación de diverso tipo entre factores neuropsicológicos, procesos cognitivos y procesos de la lectura en los niños de $2 .{ }^{\text {do }}, 3 .{ }^{\text {er }}$ y $4 .{ }^{\text {to }}$ grado de primaria pertenecientes a dos niveles socioeconómicos diferentes en el Callao.

\section{Hipótesis específicas:}

$\mathrm{H}_{1}$ : A mayor rendimiento en la prueba neuropsicológica, mayor rendimiento en las prueba de inteligencia y de lectura, en los niños de $2 .{ }^{\text {do }}, 3 .^{\text {er }}$ y $4 .{ }^{\text {to }}$ grado de primaria del Callao en la muestra total.

$\mathrm{H}_{2}$ : A mayor rendimiento en la prueba neuropsicológica, mayor rendimiento en las prueba de inteligencia y de lectura, en los niños de $2 .{ }^{\text {do }}, 3 .{ }^{\text {er }}$ y $4 .{ }^{\text {to }}$ grado de primaria del Callao pertenecientes al nivel socio-económico medio- alto: $\mathrm{B}$

$\mathrm{H}_{3}$ : A mayor rendimiento en la prueba neuropsicológica, mayor rendimiento en las prueba de inteligencia y de lectura, en los niños de $2 .{ }^{\text {do }}, 3 .^{\text {er }}$ y $4 .{ }^{\text {to }}$ grado de primaria del Callao pertenecientes al nivel socio-económico bajo: $\mathrm{E}$

$\mathrm{H}_{4}$ : Existe un buen rendimiento en factores neuropsicológicos, en los niños del $2 .{ }^{\text {do }}, 3$. $^{\text {er }}$ y $4 .{ }^{\text {to }}$ grado de primaria del Nivel socio económico medio- alto $\mathrm{B}$ y un bajo rendimiento en los niños del Nivel socio económico bajo $\mathrm{E}$.

$\mathrm{H}_{5}$ : Existe un buen rendimiento en inteligencia y procesos cognitivos, en los niños del $2 .^{\text {do }}$, 3 er $^{\text {er }} 4 .{ }^{\text {to }}$ grado de primaria del Nivel socio económico medio- alto B y un bajo rendimiento en los niños del Nivel socio económico bajo $\mathrm{E}$.

$\mathrm{H}_{6}$ : Existe un buen rendimiento en procesos de la lectura, en los niños del $2 .{ }^{\text {do }}, 3 .^{\text {er }}$ y $4 .^{\text {to }}$ grado de primaria del Nivel socio económico medio- alto B y un bajo rendimiento en los niños del Nivel socio económico bajo E.

\section{MÉTODO}

Se trató de una investigación descriptiva correlacional (Sánchez C. et al, 1984, 2004). La población estuvo constituida por los niños de segundo a cuarto grado de primaria, de Instituciones Educativas privadas perteneciente al nivel socioeconómicos: medio alto : B del distrito de Bellavista; y niños del nivel socio-económico muy bajo: E; del distrito de Ventanilla, Callao.

La muestra se escogió con criterio no probabilístico, de carácter intencional.

Tabla 1. Características generales de la muestra

\begin{tabular}{|c|c|c|c|c|c|c|c|}
\hline \multirow{2}{*}{ Escuela } & \multirow{2}{*}{ Tipo } & \multirow{2}{*}{ N. socioeconómico } & \multicolumn{2}{|c|}{ Varones } & \multicolumn{2}{|c|}{ Mujeres } & \multirow{2}{*}{ Total } \\
\hline & & & $\mathrm{N}$ & $\%$ & $\mathrm{~N}$ & $\%$ & \\
\hline I.E.P. América Bellavista & Privada & Medio-alto & 17 & 43.3 & 13 & 56.7 & 30 \\
\hline $\begin{array}{l}\text { I.E.N. Sagrado Corazón de } \\
\text { María Pachacútec Ventanilla }\end{array}$ & Pública & Muy bajo & 13 & 56.7 & 17 & 43.3 & 30 \\
\hline
\end{tabular}




\section{Instrumentos}

Se aplicaron los siguientes instrumentos:

1. Evaluación Neuropsicológica Infantil breve, de Quintanar-Solovieva (2004), prueba que se tomó en forma individual y que evaluaba factores neuropsicológicos. Su validez de contenido, en base a criterio de jueces utilizándose el Coeficiente de validez de Aiken: $\mathrm{V}=(\mathrm{X}-\mathrm{l}) / \mathrm{k}$, fue: $\mathrm{V}$ de 0.80 lo cual indica concordancia entre los jueces respecto a la validez de la prueba.

Posee adecuada Confiabilidad: 0.57, según el coeficiente alfa de Cronbach.

2. Test de Inteligencia WISC-IV de Wechsler (2006): Prueba de Inteligencia elaborada en 1949, revisada por Wechsler en el año 1973. Para nuestro estudio se usó la versión en español del test WISC. IV. Su Fiabilidad es adecuada. Los coeficientes de consistencia interna calculados con las fórmulas de Guilford (1954) y Nunnally y Bertstein (1994) sobrepasan en cada subtest el valor de: 0,72

Se hicieron estudios de validez, correlacionando con otras medidas arrojadas por tests similares: Raven, WISC III. Con la primera se halló correlación de 0,64 y con la segunda, de acuerdo a los índices: Comprensión verbal, Razonamiento perceptivo, Memoria de trabajo y Velocidad de procesamiento, la correlación oscila entre 0,72 (memoria de trajajo/ dígitos) y 0,89 (CIT/CIT).

3. Prueba de evaluación de lectura: PROLEC-R (2007) de Fernando Cuetos et al., que examina los procesos de identificación de letras, procesos léxicos o de reconocimiento de palabras, procesos sintácticos y procesos semánticos que intervienen en la lectura. La fiabilidad de la prueba, se estableció en base al estudio de su consistencia interna, con el coeficiente alfa de Cronbach. Los valores alcanzados fueron satisfactorios: 0.79. Respecto a la Validez del PROLEC R, se tomó en cuenta en primer término, su validez de criterio. Para ello se tomó en cuenta la opinión de los profesores respecto al rendimiento de los alumnos en lectura. Las correlaciones halladas fueron significativas al nivl $\mathrm{p}<0,001$

Para la presente investigación se realizó la correspondiente Validación en el Callao, hallándose resultados adecuados tanto en Validez como en Confiabilidad.

\section{RESULTADOS}

Verificación de hipótesis:

Existe asociación, de diverso tipo, entre factores neuropsicológicos, procesos cognitivos y procesos de la lectura en los niños de $2 .{ }^{\text {do }}, 3 .{ }^{\text {er }}$ y $4 .{ }^{\text {to }}$ grado de primaria pertenecientes a dos niveles socioeconómicos diferentes en el Callao.

Hipótesis específicas:

$\mathrm{H}_{1}$ : A mayor rendimiento en la prueba neuropsicológica, mayor rendimiento en las prueba de inteligencia y de lectura, en los niños de $2 .{ }^{\text {do }}, 3 .{ }^{\text {er }}$ y $4 .{ }^{\text {to }}$ grado de primaria del Callao en la muestra total. 
A) Se buscó establecer si existía relación entre los factores neuropsicológicos a nivel de puntuación total y el Cociente de Inteligencia (WISC- IV). Se encontró una correlación inversa estadísticamente significativa -0.45 entre dichos rendimientos. Hay que indicar que en la Prueba neuropsicológica, dado que se califica de acuerdo a los errores, quienes puntúan más bajo, están mejor.

B) Igualmente, se buscó establecer si existía relación entre los factores neuropsicológicos a nivel de puntuación total, y el rendimiento en los diversos procesos de la lectura (PROLEC-R) a nivel de puntaje global.

Se encontró una correlación inversa estadísticamente significativa -0.42 entre dichos rendimientos, es decir los niños que presentaban mejor rendimiento en la Prueba de Quintanar: factores neuropsicológicos, presentaban mejores puntajes a nivel del PROLEC-R.

C) Por último, se buscó establecer la correlación entre el CI total y el rendimiento en el PROLEC-R (puntuación global), hallándose una correlación directa estadísticamente significativa 0.52 entre dichos rendimientos, es decir los niños que presentaban los mejores puntajes en términos CI total tendían a presentar mejores puntajes a nivel del PROLEC- R.

$\mathrm{H}_{2}$ : A mayor rendimiento en la prueba neuropsicológica, mayor rendimiento en las prueba de inteligencia y de lectura, en los niños de $2 .{ }^{\mathrm{do}}, 3 .{ }^{\text {er }} \mathrm{y} 4 .{ }^{\text {to }}$ grado de primaria del Callao pertenecientes al nivel socioeconómico medio-alto: B

El análisis correlacional entre las variables estudiadas se realizó a través del estadístico no paramétrico Rho de Spearman. Los resultados se muestran en las tablas siguientes.

\section{- Correlaciones significativas las que están sombreadas en gris}

El análisis correspondiente, nos indica que en el nivel socioeconómico medio alto B, se halló correlación inversa significativa entre el factor neuropsicológico: Organización cinética del movimiento y los procesos cognitivos: Razonamiento perceptivo e Inteligencia total. También entre el factor neuropsicológico Memoria audio- verbal y el proceso de la lectura: procesos semánticos.

De otro lado, se halló correlación directa muy significativa entre los factores cognitivos de Comprensión verbal y el Razonamiento perceptivo y la Inteligencia total. También entre Razonamiento perceptivo y la Inteligencia total, y Memoria de trabajo e Inteligencia total.

Así también se halló correlación muy significativa directa entre Comprensión verbal y los procesos de la lectura: gramaticales y semánticos.

Y finalmente, entre los propios procesos de la lectura, correlación muy significativa entre Identificación de letras y procesos léxicos y gramaticales; y procesos léxicos con los procesos gramaticales.

$\mathrm{H}_{3}$ : A mayor rendimiento en la prueba neuropsicológica, mayor rendimiento en las prueba de inteligencia y de lectura, en los niños de $2 .{ }^{\text {do }}, 3 .{ }^{\text {er }}$ y $4 .{ }^{\text {to }}$ grado de primaria del Callao pertenecientes al nivel socioeconómico bajo: E. 
Ricardo Canales G.

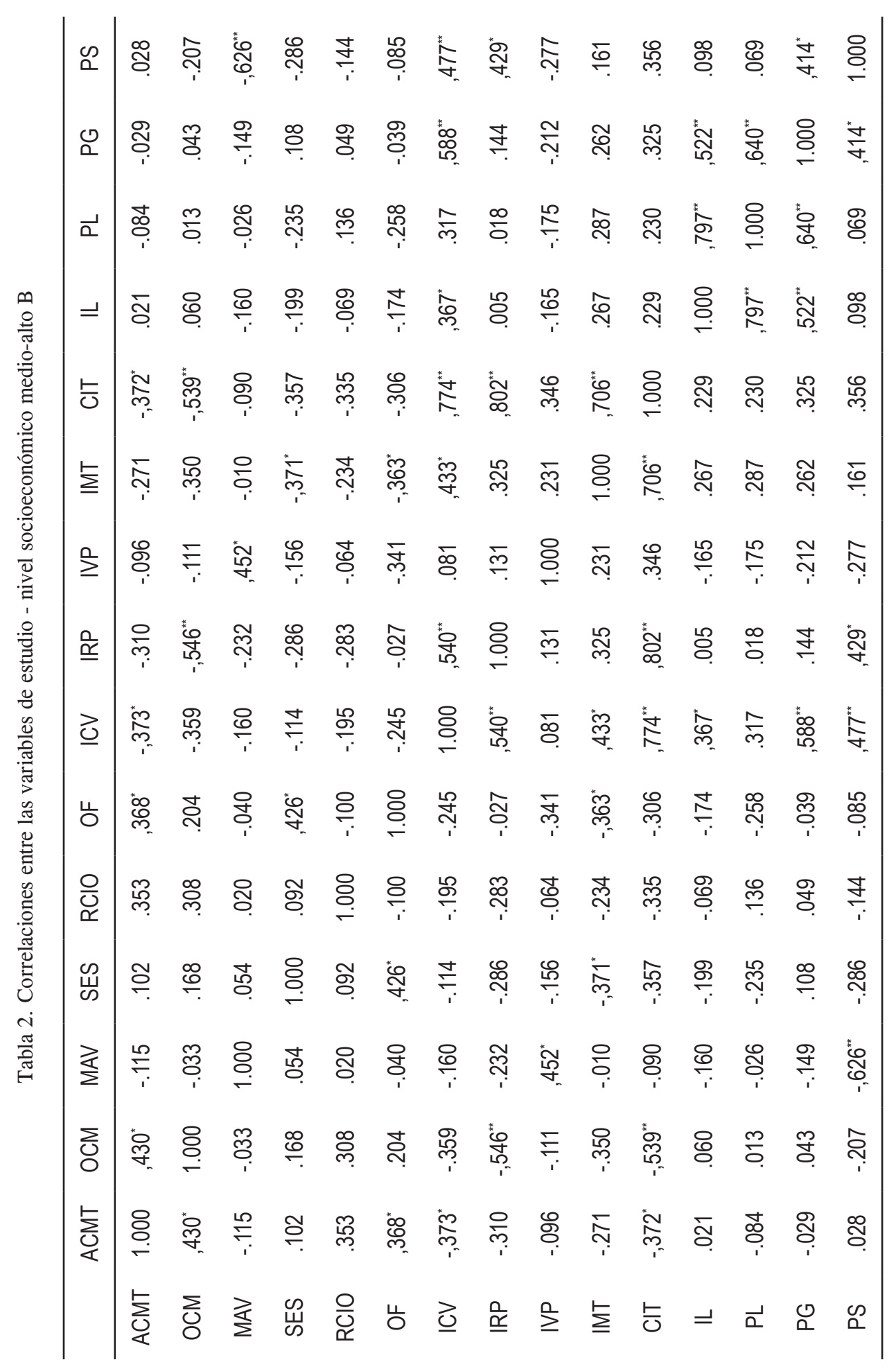


ASOCIACIÓN ENTRE FACTORES NEUROPSICOLÓGICOS, PROCESOS COGNITIVOS Y NIVELES DE LECTURA...

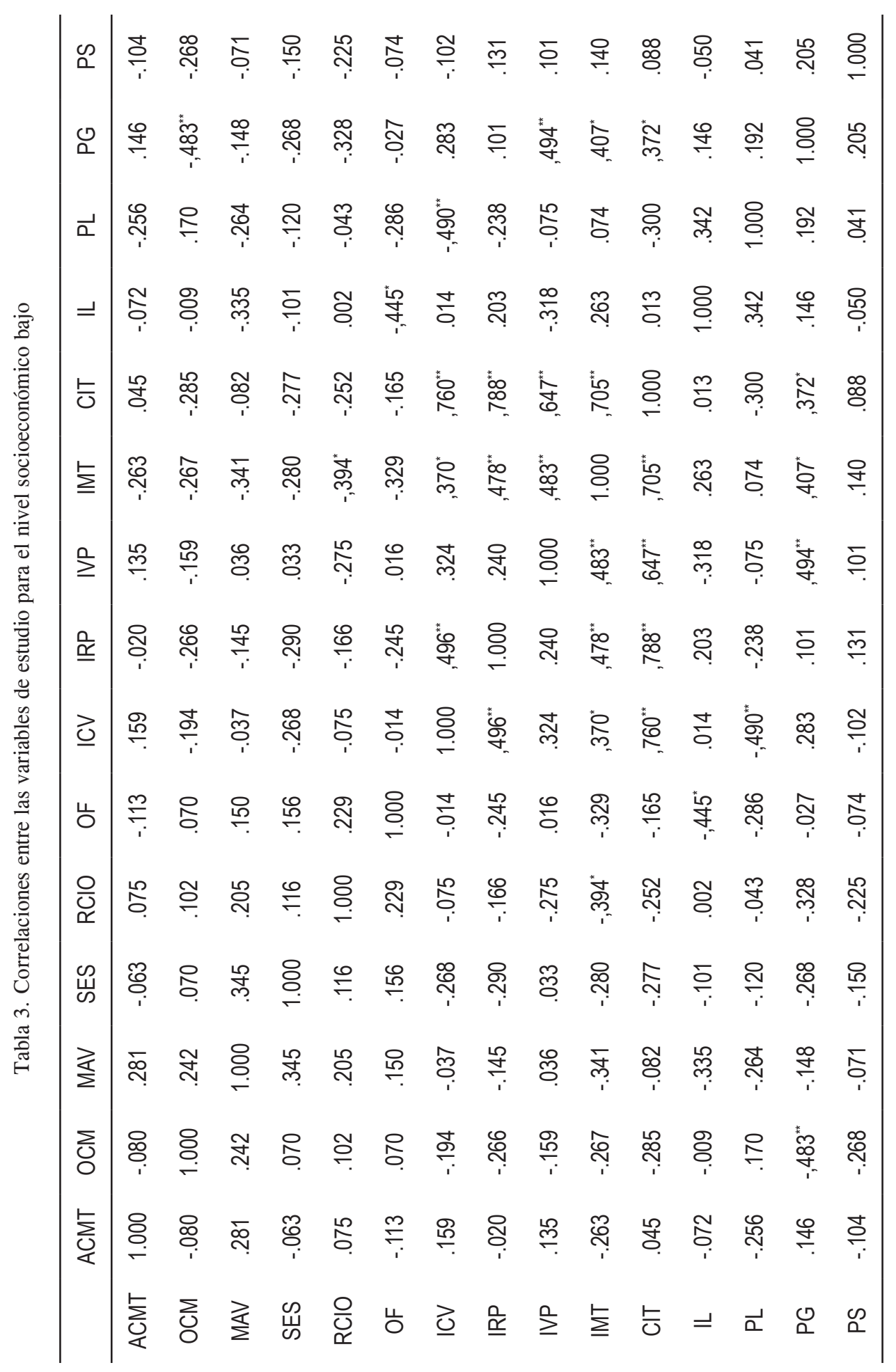




\section{- Correlaciones significativas las que están sombreadas en gris}

El análisis correspondiente, nos indica que en el nivel socioeconómico muy bajo E, se halló correlación inversa significativa entre el factor neuropsicológico: organización cinética del movimiento, y proceso psicológico de la lectura: proceso gramatical.

Respecto a la relación entre procesos cognitivos se observa correlación directa muy significativa entre Comprensión verbal y Razonamiento perceptivo, así como entre Comprensión verbal e Inteligencia total. Y una correlación inversa significativa entre Comprensión verbal y el proceso psicológico de la lectura: procesos léxicos.

También se observa correlación directa muy significativa entre Razonamiento perceptivo y Memoria de trabajo e Inteligencia total. De igual modo entre Velocidad de procesamiento y Memoria de trabajo e Inteligencia total, así como entre Velocidad de procesamiento y el proceso psicológico de la lectura: proceso gramatical.

Por último, se encuentra correlación directa muy significativa entre Memoria de trabajo y la Inteligencia total.

H4: Existe un buen rendimiento en factores neuropsicológicos, en los niños del $2 .{ }^{\text {do }}, 3 .{ }^{\text {er }}$ y $4 .^{\text {to }}$ grado de primaria del Nivel socio económico medio- alto B y un bajo rendimiento en los niños del Nivel socio económico bajo E.

Tabla 4. Rendimiento en factores neuropsicológicos para el 2. ${ }^{\text {do }}$ grado de Educación Primaria

\begin{tabular}{lcccc} 
& \multicolumn{2}{c}{ NSE B } & \multicolumn{2}{c}{ NSE E } \\
\multicolumn{1}{c}{ Factores neuropsicológicos } & Media & DS & Media & DS \\
\hline 1. Analizador cinestésico y memoria táctil & 14,27 & 1,009 & 14,00 & 1,826 \\
2. Organización cinética de los movimientos & 3,55 &, 688 & 4,40 & 1,776 \\
3. Memoria audioverbal y visual & 17,27 & 2,901 & 19,80 & 1,135 \\
4. Síntesis espacial simultánea & & & & 1,229 \\
5. Regulación y control e Imágenes objetales & 3,36 &, 674 & 4,20 & 1,317 \\
6. Oído fonemático & 4,45 &, 820 & 4,80 & \\
\hline
\end{tabular}

Los resultados para el $2 .^{\text {do }}$ grado indican que solo en el factor neuropsicológico de memoria audioverbal y visual los niños del nivel socioçeconómico medio- alto $\mathrm{B}$, rinden mejor. En el factor neuropsicológico de oído fonemático, en cambio, alcanzan mejor rendimiento los niños del nivel socioeconómico bajo E. 
ASOCIACIÓN ENTRE FACTORES NEUROPSICOLÓGICOS, PROCESOS COGNITIVOS Y NIVELES DE LECTURA...

Tabla 5. Rendimiento en factores neuropsicológicos para el $3^{\circ}$ grado de Educación primaria

\begin{tabular}{lcccc}
\hline \multicolumn{1}{c}{ Factores neuropsicológicos } & \multicolumn{2}{c}{ NSE B } & \multicolumn{2}{c}{ NSE E } \\
& Media & DS & Media & DS \\
\hline 1. Analizador cinestésico y memoria táctil & 14,00 & 1,826 & 14,33 & 1,871 \\
2. Organización cinética de los movimiento & 4,40 & 1,776 & 4,22 &, 833 \\
3. Memoria audioverbal y visual & 19,80 & 1,135 & 18,33 & 2,598 \\
4. Síntesis espacial simultánea & & & & \\
5. Regulación y control e Imágenes objetales & 4,20 & 1,229 & 3,11 &, 333 \\
6. Oído fonemático & 4,80 & 1,317 & 4,56 &, 882 \\
\hline
\end{tabular}

Los resultados para el 3 er $^{\text {er }}$ grado indican que en el factor neuropsicológico de oído fonemático los niños del nivel socioeconómico medio-alto $\mathrm{B}$, rinden mejor, mientras que en el factor de síntesis espacial simultánea, el mejor rendimiento es para los niños nivel socio-económico E.

Tabla 6. Rendimiento en factores neuropsicológicos para el 4. ${ }^{\text {to }}$ grado de Educación primaria

\begin{tabular}{lcccc}
\hline & \multicolumn{2}{c}{ NSE B } & \multicolumn{2}{c}{ NSE E } \\
Factores neuropsicológicos & Media & DS & Media & DS \\
\hline 1. Analizador cinestésico y memoria táctil & 13,20 & 1,135 & 15,00 & 2,309 \\
2. Organización cinética de los movimientos & 3,60 &, 699 & 4,50 & 1,354 \\
3. Memoria audioverbal y visual & 18,00 & 3,162 & 18,00 & 2,404 \\
4. Síntesis espacial simultánea & & & & \\
5. Regulación y control e imágenes objetales & 3,40 &, 699 & 3,90 & 1,101 \\
6. Oído fonemático & 4,30 &, 675 & 4,60 &, 843 \\
\hline
\end{tabular}

Los resultados para el $4 .^{\text {to }}$ grado indican que son los niños del nivel socioeconómico bajo E., los que rinden mejor, ahora en el factor neuropsicológico de oído fonemático. Los niños del nivel socioeconómico medio $\mathrm{B}$, alcanzan un mejor 
rendimiento en el factor Analizador cinestésico y memoria táctil. En el resto no hay mayor diferencia.

$\mathrm{H}_{5}$ : Existe un buen rendimiento en inteligencia y procesos cognitivos, en los niños del $2 .{ }^{\text {do }}, 3 .^{\text {er }}$ y $4 .^{\text {to }}$ grado de primaria del Nivel socioeconómico medio-alto B y un bajo rendimiento en los niños del Nivel socioeconómico bajo E.

Los resultados para el 2. ${ }^{\text {do }}$, grado indican que los niños del nivel socioeconómico medio B, alcanzan los mejores rendimientos en inteligencia total y en los índices de componente verbal, organización perceptual, memoria de trabajo y velocidad de procesamiento.

Los resultados para el 2. ${ }^{\text {do }}$, grado indican que los niños del nivel socioeconómico medio B, alcanzan los mejores rendimientos en inteligencia total y en los índices de componente verbal, organización perceptual y memoria.

Los resultados para el $4{ }^{\text {to }}$ grado indican que los niños del nivel socioeconómico medio B, alcanzan los mejores rendimientos en inteligencia total y en los índices de componente verbal, organización perceptual y memoria.

$\mathrm{H}_{6}$ : Existe un buen rendimiento en procesos de la lectura, en los niños del 2 . $^{\text {do }}$, $3 .^{\text {er }}$ y $4 .{ }^{\text {to }}$ grado de primaria del Nivel socioeconómico medio-alto B y un bajo rendimiento en los niños del Nivel socioeconómico bajo E.

Los resultados para el 2. do grado indican que los niños del nivel socioeconómico medio B obtienen los mejores puntajes en la identificación de letras, los procesos léxicos, los procesos gramaticales y los procesos semánticos.

Los resultados para el $3 .{ }^{\text {er }}$ grado indican que existen diferencias significativas a favor del grupo proveniente del nivel socioeconómico medio en la identificación de letras, los procesos léxicos y los procesos gramaticales.

Los resultados para el $4 .^{\text {to }}$ grado indican que en este caso son los niños del nivel socioeconómico bajo E que rinden mejor en el proceso de la lectura de Identificación de letras; mientras que los niños del nivel socioeconómico medio $\mathrm{B}$, lo hacen mejor en procesos léxicos y los procesos semánticos.

\section{DISCUSIÓN}

Analizando los resultados del presente estudio, respecto a la asociación y el tipo de la misma, entre factores neuropsicológicos, procesos cognitivos y procesos de la lectura se encontró entre los dos primeros, una correlación inversa de: - 0, 45 la cual es estadísticamente significativa. Esto quiere decir, que el buen desarrollo de factores neuropsicológicos como la capacidad de planeación y control, organización cinestésica del movimiento, praxias y gnosias, percepción visual, memoria audio-verbal y oído fonemático (Akhutina, 1999, Quintanar, 2004) correlaciona con un buen desarrollo de la inteligencia en los niños de la muestra total estudiada.

Cuando se desagrega este resultado, entre los dos grupos socioeconómicos, se observa que en el nivel socioeconómico medio alto B, la correlación es mayor: 
-0,49 mientras en el nivel socioeconómico bajo E, la correlación es: - 0,35 , lo cual indicaría que en los primeros niños el desarrollo de sus factores neuropsicológicos muestra una tendencia más clara a concordar con los procesos cognitivos, tales como la comprensión verbal, el razonamiento perceptivo, la memoria de trabajo, la velocidad de procesamiento y la inteligencia general. En cambio, en el segundo grupo, dicha tendencia es menos clara.

En este punto es importante señalar que en los estudios desarrollados en México por Quintanar y cols. $(1997,2002)$ se apreció que personas de zonas rurales (analfabetas, primaria incompleta) obtenían un bajo rendimiento en las pruebas neuropsicológicas, lo cual correlacionaba con bajo nivel de educación y de desarrollo cognitivo.

Respecto a la relación entre factores neuropsicológicos y lectura, al hacer el análisis de correlaciones global se encontró una correlación de: - 0,42 la cual es, también estadísticamente significativa. Esto quiere decir que los niños con mayor nivel de desarrollo en las habilidades para la planeación y control, organización cinestésica del movimiento, praxias y gnosias, percepción visual, memoria audio-verbal y oído fonemático, correlaciona con una mayor habilidad en el aprendizaje de la lectura, lo cual implica un mayor desarrollo en los procesos perceptivos, léxicos, sintácticos y semánticos de la misma. En sentido contrario, los niños más deficientes en los factores neuropsicológicos mencionados, rinden más bajo en lectura (entre ellos habría un buen número de niños con Retardo lector o posible Dislexia).

Este dato, de algún modo se relaciona con lo hallado por Manga (1991) cuando comparando lectores normales y niños disléxicos, luego de aplicar la prueba Luria- DNI, encontró que en los diferentes factores examinados: Motricidad, audición, tacto y cinestesia, visión, habla receptiva, habla expresiva, lecturaescritura, aritmética y memoria, los disléxicos alcanzaban puntajes más bajos en el conjunto de dichos factores.

Por último se encontraron correlaciones entre los componentes cognitivo e inteligencia y los procesos de la lectura. A lo hallado en el nivel socioeconómico $\mathrm{B}$, es decir la relación entre el Componente verbal y la Identificación de letras, Procesos gramaticales y semánticos, y en el grupo socioeconómico E, la relación significativa entre Velocidad de procesamiento con los Procesos gramaticales, se añadió el hecho de encontrarse correlación global directa de: 0,52, la cual es estadísticamente significativa.

Esto quiere decir que los niños que han desarrollado de mejor modo sus Componentes verbales (capacidad de establecer relaciones lógicas, vocabulario, información, etc.), su Organización perceptiva (formulación de estrategias, solución de problemas con material visual y concreto), su Velocidad de procesamiento de la información (el factor tiempo) y la Memoria de trabajo, estarían en mejores condiciones de desarrollar los diversos mecanismos y procesos que implica la lectura (identificación de letras, reconocimiento léxico, procesos sintácticos y semánticos) tanto en los aspectos de la decodificación como de la comprensión. 
Tratando de relacionar lo hallado con aspectos del funcionamiento neuropsicológico, habría que indicar, siguiendo a Luria, que el llamado factor secuencial, que alude a la forma en que el cerebro procesa la información que proviene del medio externo en forma discreta, secuencial (es característico que la información lingüística se procesa en dicha dimensión), sería un punto crítico en el funcionamiento mental de los niños del nivel socioeconómico bajo E. De otro lado, la investigación psicológica y neuropsicológica actual ha precisado la importancia que para aprender a leer, tiene la conciencia fonológica (Vellutino 1983, 1991, Badian 1991, Etchepareborda 2001) y dicha función psicolingüística compleja tiene su base en la estructura del planum temporale y el giro angular izquierdo.

En relación a las otras hipótesis específicas, que buscan examinar los rendimientos considerando la pertenencia a diferente nivel socio- económico se halló en el nivel socio económico B (medio alto), correlación inversa muy significativa entre el factor neuropsicológico: Organización cinética del movimiento y los factores cognitivos: Razonamiento perceptivo e Inteligencia total. Este hallazgo indicaría que los niños que han desarrollado una mayor capacidad en los aspectos de la programación y regulación de la acción (en la prueba neuropsicológica, se examinan las praxias) rinden mejor en las tareas de diseño y ejecución de estrategias solucionadoras de problemas (Diseño de cubos, prueba de matrices en el WISCIV), lo cual a su vez correlaciona con el Cuociente de Inteligencia total.

También se observa correlación inversa muy significativa, entre el factor neuropsicológico Memoria audio- verbal y los procesos semánticos de la lectura. Este hallazgo permitiría establecer una relación entre la capacidad de almacenar información, por vía visual y auditiva en el corto plazo (incluso con interferencia) con la memoria semántica (muy importante para la lectura).

De otro lado, se halló correlación directa muy significativa entre los factores cognitivos de Comprensión verbal y Razonamiento perceptivo e Inteligencia, así como entre Comprensión verbal y los procesos de la lectura: Gramaticales y Semánticos a nivel muy significativo.

En el nivel socio económico E (muy bajo), se encontró correlación inversa muy significativa entre el factor neuropsicológico: Organización cinética del movimiento (examinado a través de ejecución de diversas praxias) y proceso gramatical de la lectura. Asimismo se encontró correlación inversa significativa entre el factor neuropsicológico de Regulación, control e identificación de imágenes objetales con la Memoria de trabajo. Ello indicaría que los niños que han desarrollado mayor habilidad para procesar información verbal y de acuerdo a ello, regulan su conducta; mostrarían un mejor desarrollo en aquel tipo de memoria que nos permite no solo el almacenamiento de la información en el corto plazo, sino también el monitoreo de los otros procesos cognitivos. 
ASOCIACIÓN ENTRE FACTORES NEUROPSICOLÓGICOS, PROCESOS COGNITIVOS Y NIVELES DE LECTURA...

\section{CONCLUSIONES}

1. Se estableció asociación de diferente tipo entre los factores neuropsicológicos, los procesos cognitivos e inteligencia, y procesos de la lectura, en los niños de la muestra examinada.

2. Se halló que a mayor rendimiento en la prueba neuropsicológica, mayor rendimiento en las prueba de inteligencia y de lectura, en los niños de $2 .{ }^{\mathrm{do}}, 3 .^{\text {er }}$ y $4 .{ }^{\text {to }}$ grado de primaria del Callao en la muestra total.

3. Se halló relación entre los factores neuropsicológicos, y la inteligencia en los niños de $2 .^{\text {do }}, 3 .^{\text {er }}$ y $4 .^{\text {to }}$ grado de primaria del Callao examinados.

4. Se halló relación entre los factores neuropsicológicos, y los procesos de lectura en los niños de $2 .{ }^{\text {do }}, 3 .^{\text {er }}$ y $4 .{ }^{\text {to }}$ grado de primaria del Callao examinados.

5. Se halló relación entre Inteligencia y el rendimiento en lectura en los niños de $2 .{ }^{\text {do }}, 3$. $^{\text {er }}$ y $4 .{ }^{\text {to }}$ grado de primaria del Callao examinados.

6. En los niños de $2 .{ }^{\text {do }}, 3 .^{\text {er }}$ y $4 .{ }^{\text {to }}$ grado de primaria del Callao pertenecientes al nivel socioeconómico medio-alto: $\mathrm{B}$, se encontró que a mayor rendimiento en factores neuropsicológicos (organización cinética del movimiento y Memoria audio-verbal) hubo un mayor rendimiento en los procesos de lectura, particularmente semánticos.

7. En los niños de $2 .{ }^{\text {do }}, 3{ }^{\text {er }}$ y $4 .{ }^{\text {to }}$ grado de primaria del Callao pertenecientes al nivel socio-económico muy bajo: E, se encontró que a mayor rendimiento en factores neuropsicológicos (organización cinética del movimiento y Regulación, control e identificación de imágenes objetales) hubo un mayor rendimiento en el proceso cognitivo Memoria de trabajo y los procesos gramaticales de la lectura. Así también entre Oído fonemático y el proceso de Identificación de letras en la lectura.

8. En 2. do Grado del nivel socioeconómico medio-alto B se halló buen rendimiento en los factores neuropsicológicos de memoria audio-verbal y visual, así como en menor grado en el resto de factores. En el 3. ${ }^{\text {er }}$ grado rindieron mejor en oído fonemático y en $4 .^{\text {to }}$ grado en Analizador cinestésico y memoria táctil. En dicho grado, los niños del nivel socioeconómico bajo E, rinden mejor en Oído fonemático.

9. Los niños del $2 .{ }^{\text {do }}, 3$ er $^{\text {er }} 4 .^{\text {to }}$ grado de primaria del nivel socioeconómico medio-alto $\mathrm{B}$ rindieron mejor en procesos cognitivos e inteligencia, mientras que los niños del Nivel socioeconómico bajo E, obtuvieron un bajo rendimiento.

10. Los niños del $2 .^{\text {do }}, 3$ er $^{\text {er }} 4 .^{\text {to }}$ grado de primaria del nivel socioeconómico medio-alto B obtuvieron un buen rendimiento en el conjunto de los procesos psicológicos de la lectura, mientras que los niños del Nivel socioeconómico bajo E, obtuvieron un bajo rendimiento, a excepción del proceso de Identificación de letras.

11. Los instrumentos utilizados se demostraron como confiables y útiles para el examen de las variables neuropsicológicas, cognitivas y de lectura planteadas. 


\section{REFERENCIAS BIBLIOGRÁFICAS}

Bravo, L. (2004). Lenguaje Escrito y Dislexia. Santiago de Chile. Ediciones Universidad Católica de Chile.

Canales, R.(2005). Procesos cognitivos y estrategias psicolingüísticas que intervienen en la lectura comprensiva: diseño y ejecución de un programa experimental en niños con problemas de aprendizaje. Tesis Doctoral en Psicología. Lima. UNMSM.

Canales, R. (2007). Comprensión lectora y problemas de aprendizaje. Un enfoque cognitivo. Lima. Edic. CONCYTEC.

Canales, R. y Velarde, E. (2008). La lectura en el Perú: Drama y esperanza. Lima. Edic. Universidad Enrique Guzmán y Valle. La Cantuta.

Cuetos, F. (2008). Psicología de la lectura. Madrid. Edic. Wolters Kluve.

González, R. (2006). Problemas psicolinguísticos en el Perú. Lima. Edic. N. Reátegui.

Luria, A. (1979). El cerebro en acción. Barcelona. Edit. Fontanella.

Manga, D. (1991). Neuropsicología de la edad escolar. Aplicaciones de la teoría de Luria a niños a través de la Batería LURIA- DNI. Madrid. Edic. Visor.

Molina García, S. (1998). El fracaso en el aprendizaje escolar. Málaga. Edic. Aljibe.

Ortiz, P. (2002). Lenguaje y habla personal. Lima. Fondo Edit. UNMSM. Prueba PISA. Programa Internacional de Evaluación de estudiantes (Programme for Internacional Student Assesment). En: www.pisa.oecd.org/index.htm

Quintanar, L. , Solovieva, Y. (2004). Evaluación Neuropsicológica Infantil. Lima. Perú Edic. Libro Amigo.

Sánchez, H., Reyes, C. (2002). Metodología y diseños en la investigación científica. Lima. Perú. Edic. Universidad Ricardo Palma.

Velarde, E. (2001). Relación entre la conciencia fonológica y el nivel de decodificación y comprensión lectora en niños de 8 años del $3{ }^{\text {er }}$ grado de primaria de dos niveles socioeconómicos del Cercado del Callao. Tesis para obtener el grado de Magíster en Educación. Lima. UNIFE.

Velarde C. Esther.(2009). Elaboración y aplicación de un programa metafonológico en niños(as) de 8 a 10 años de $3{ }^{\text {er }}$ y $4 .{ }^{\text {to }}$ grado de primaria del cercado del Callao". Tesis Doctoral en Educación. UNMSM. Lima.

Velasco, R. y Castro, C. (2002). Trastornos neuropsicológicos en niños con epilepsia. Lima. Revista de Neuropsiquiatría. 65

Wechsler, D. (2006). WISC-IV Escala para la inteligencia de Wechsler para niños IV. Manual técnico de interpretación. Madrid. Edic. TEA 